\title{
FPGA Implementation of Low Power and High Speed Vedic Multiplier using Vedic Mathematics.
}

\author{
Anju \& V.K. Agrawal, \\ Department of ECE, I.M.S. Engineering Collage, NH-24, Adhyatmik Nagar, Ghaziabad-201009, U.P., India
}

\begin{abstract}
A high speed processor depends greatly on the multiplier as it is one of the key hardware blocks in most digital signal processing system as well as in general processors. This paper proposes the design of $8 \times 8$ bit Vedic multiplier based on vertical and crosswise structure of Ancient Indian Vedic Mathematics. The proposed architecture is for two 8-bit numbers, the multiplier and multiplicand each arc grouped as 4-bit numbers; so that it decomposes into $4 \times 4$ multiplication modules. This gives chance for modular design where smaller blocks can be used to design the bigger one. Further, the VHDL coding of Urdhava Tiryakbhyam sutra for $8 \times 8$ bits multiplication and their FPGA implementation by Xilinx Synthesis Tool have been done.
\end{abstract}

Keywords- FPGA, Urdhua Tiryagbhyam sutra, Vedic mathematics, Xilinx.

\section{Introduction}

With the latest advancement of VLSI technology the demand for portable and embedded digital signal processing (DSP) systems has increased efficiently. Multipliers are key components of many high performance systems such are FIR filters, Microprocessors, Digital Signal Processors etc. For higher order multiplications, a large number of adders or components are used. The speed of the multiplier depends on the number of partial product and the speed of adder.

Vedic mathematics was reconstructed from 'Vedas' by Sri Bharti Krishna Tirthaji (1884-1960) after his eight years of research on Vedas [1]. According to him, Vedic mathematics is mainly focused on sixteen very important principles or word-formulae which are termed as sutras. In this paper after a gentle introduction of Urdhavatriyakb-hyam sutra, multiplier architecture is discussed and is illustrated with two 8-bit numbers.

The multiplier and multiplicand, each are grouped as 4-bit numbers so that it decomposes into $4 \times 4$ multipl-ication module. After decomposition, vertical and cross-wise is applied to carry out the multiplication on first $4 \times 4$ multiply module[2]. The multiplier based on this sutra has the advantage that as the number of bits increases, gate delay and area increase very slowly as compared to other conventional multipliers. Vedic Sutras are applied to binary multipliers using carry save adders. The Vedic multiplier which is implemented by us and is discussed in this paper, performs partial product generation and addition in parallel has better performance in turns of speed and area.

\section{Vedic Multiplier using 'Urdhya Tiryagbhyam Sutra}

The use of Vedic mathematics lies in the fact that it reduces the typical calculations in conventional mathematics into very simple one. This is so because the Vedic formulae are claimed to be based on the natural principles on which the human mind works. Vedic mathematics is a methodology of arithmetic rules that allow more efficient speed implementation. UrdhvaTriyakbhyam is the general formula applicable to all cases of multiplication.

'Urdhva' and 'Tiryagbhyam' words are derived from Sanskrit literature. 'Urdhva' means 'Vertically' and 'Tirya-gbhyam' means 'Crosswise'[3]. It is based on a novel concept through which the generation of all partial products can be done with the concurrent addition of these partial product.

The sutra is illustrated in Fig 1. and the hardware architecture is depicted in Fig. 2 in this example two decimal numbers $252 \times 846$ are multiplied.

The digits on the both sides of the line are multiplied and added with the carry from the previous step. This generates one of the bits of the result and a carry. This carry is added in the next step and hence the process goes on. If more than one line is there in one step, all the results are added to the previous carry. In each step, least significant bit acts as the result bit and all other bits act as carry for the next step. Initially the carry is taken to be zero. 
Example 1: $325 \times 738=239850$

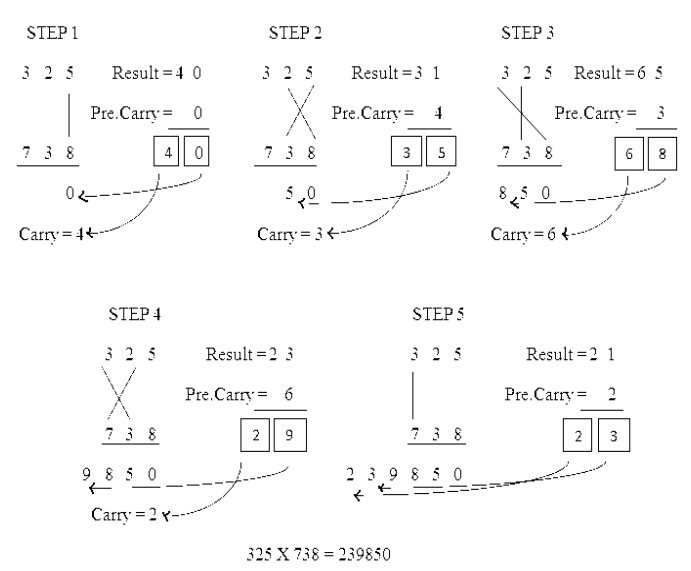

Answer: $325 \times 738=239850$.

Fig. 1 Multiplication of two decimal Numbers by Urdhava Tiryakbhyam

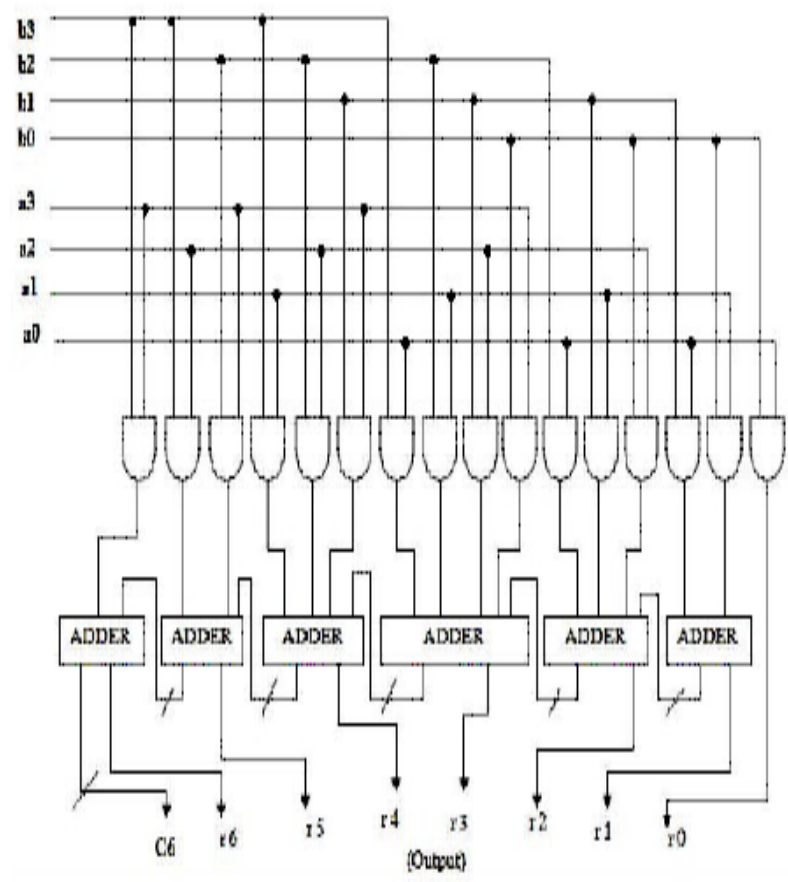

Fig. 2 Urdhava Multiplier Hardware Architechure

The 'Urdhva Tiryagbhyam' algorithm can be implemented for binary number system in the same way as decimal number system. For the multiplication algorithm, let us consider the multiplication of two 4 bit numbers $a_{3} a_{2} a_{1} a_{0}$ and $b_{3} b_{2} b_{1} b_{0}$. As the result of this multiplication of two 4 bit, we express it as $c_{7} r_{6} r_{5} r_{4} r_{3} r_{2} r_{1} r_{0}$.

Least significant bit $r_{0}$ is obtained by multiplying the least significant bits of the multiplicand and the multiplier as shown in Fig 3. The digits on both sides of the lines are multiplied and added with the carry from the previous step [4-7]. This generates on the bits of the result $\left(r_{n}\right)$ and a carry $\left(c_{n}\right)$. This carry is added in the next step and thus the process goes on.

Thus the following expressions (I) to (7) are derived:

$$
\begin{aligned}
& r_{0}=a_{0} b_{0} \\
& c_{1} r_{1}=a_{1} b_{0}+a_{0} b_{1} \\
& c_{2} r_{2}=c_{1}+a_{2} b_{0}+a_{1} b_{1}+a_{0} b_{2} \\
& c_{3} r_{3}=c_{2}+a_{3} b_{0}+a_{2} b_{1}+a_{1} b_{2}+a_{0} b_{3} \\
& c_{4} r_{4}=c_{3}+a_{3} b_{1}+a_{2} b_{2}+a_{1} b_{3} \\
& c_{5} r_{5}=c_{4}+a_{3} b_{2}+a_{2} b_{3}
\end{aligned}
$$




$$
\mathrm{c}_{6} \mathrm{r}_{6}=\mathrm{c}_{5}+\mathrm{a}_{3} \mathrm{~b}_{3}
$$

With $\mathrm{c}_{6} \mathrm{r}_{6} \mathrm{r}_{5} \mathrm{r}_{4} \mathrm{r}_{3} \mathrm{r}_{2} \mathrm{r}_{1} \mathrm{r}_{0}$ being the final product. Partial products are calculated in parallel and hence the delay involved is just the time it takes for the signal to propagate through the gates.

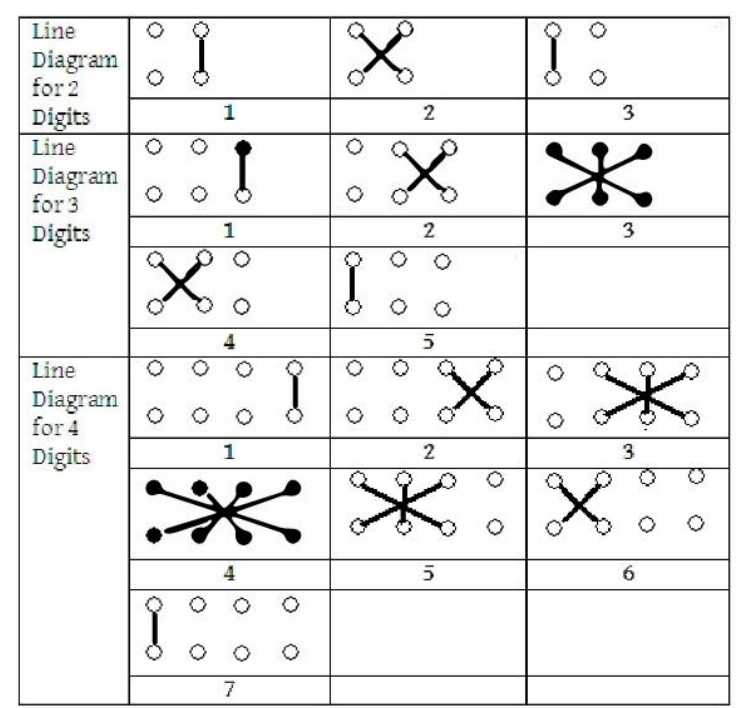

Fig.3. Line Diagram for Urdahva Multiplication of 2,3 and 4 digits

\section{The Proposed Multiplier Architecture}

The hardware architecture of $4 \times 4$ and $8 \times 8$ bit Vedic multiplier module are displayed in the below sections. Here UrdhvaTiryagbhyam (vertically and crosswise) sutra is used to propose such architecture for the multiplication of two binary numbers. The beauty of Vedic multiplier is that here partial product generations and additions are done concurrently. Hence it is well adapted to parallel processing. The feature makes it more attractive for binary multiplications. This in turn reduces delay, which is the primary motivations behind this work.

\subsection{Vedic Multiplier for $4 \times 4$ bit module.}

The $4 \times 4$ bit Vedic multiplier module is implemented using four $2 \times 2$ bit Vedic multiplier modules. Let's analyze $4 \times 4$ multiplications say $A=A_{3} A_{2} A_{1} A_{0}$ and $B=B_{3} B_{2} B_{1} B_{0}$. The output line for the multiplication results is $=S_{7} S_{6} S_{5} S_{4} S_{3} S_{2} S_{1} S_{0}$. Let's divide $A$ and $B$ into two parts say $A_{3} A_{2} \& A_{1} A_{0}$ for $A$ and $B_{3} B_{2} \& B_{1} B_{0}$ for $B$ using the fundamental of Vedic multiplication, taking two bit at a time and using 2 bit multiplier block, we can have the following structure for multiplication as shown in Fig. 4.

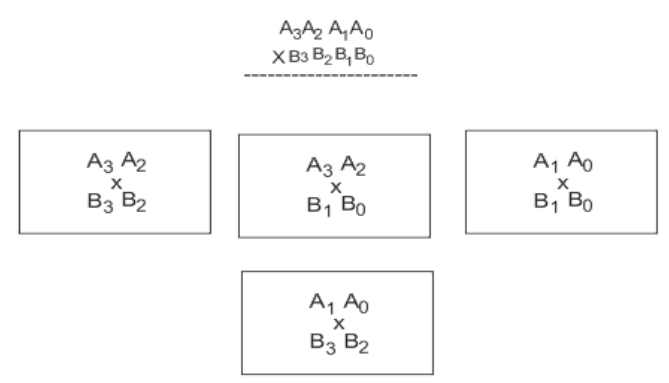

Fig 4: Algorithm of 4x4 bit Vedic Multiplier.

Each block as shown above is $2 \times 2$ bit Vedic multiplier. First $2 \times 2$ bit multiplier inputs are $\mathrm{A}_{1} \mathrm{~A}_{0}$ and $\mathrm{B}_{1} \mathrm{~B}_{0}$. The last block is $2 \times 2$ bit multiplier with inputs $\mathrm{A}_{3} \mathrm{~A}_{2}$ and $\mathrm{B}_{3} \mathrm{~B}_{2}$. The middle one shows two $2 \times 2$ bit multiplier with inputs $A_{3} A_{2} \& B_{1} B_{0}$ and $A_{1} A_{0} \& B_{3} B_{2}$. So the final result of multiplication, which is of 8 bit, $\mathrm{S}_{7} \mathrm{~S}_{6} \mathrm{~S}_{5} \mathrm{~S}_{4} \mathrm{~S}_{3} \mathrm{~S}_{2} \mathrm{~S}_{1} \mathrm{~S}_{0}$. to understand the concept, the Block diagram of $4 \times 4$ bit Vedic multiplier is shown in Fig 4.To get final product $\left(\mathrm{S}_{7} \mathrm{~S}_{6} \mathrm{~S}_{5} \mathrm{~S}_{4} \mathrm{~S}_{3} \mathrm{~S}_{2} \mathrm{~S}_{1} \mathrm{~S}_{0}\right)$ four $2 \times 2$ bit Vedic multiplier and three 4-bit Ripple-Carry (RC) adders are required. 
The proposed Vedic multipliers can be used to reduce delay. Early literature speaks about Vedic multiplier based on array multiplier structures. On the other hand, we proposed a new architecture, which is efficient in terms of speed. The arrangements of RC Adders showed in Fig. 5 helps us to reduce delay. Interestingly, 8x8 Vedic multiplier modules are implemented easily by using four 4x4 multiplier modules[8-10].

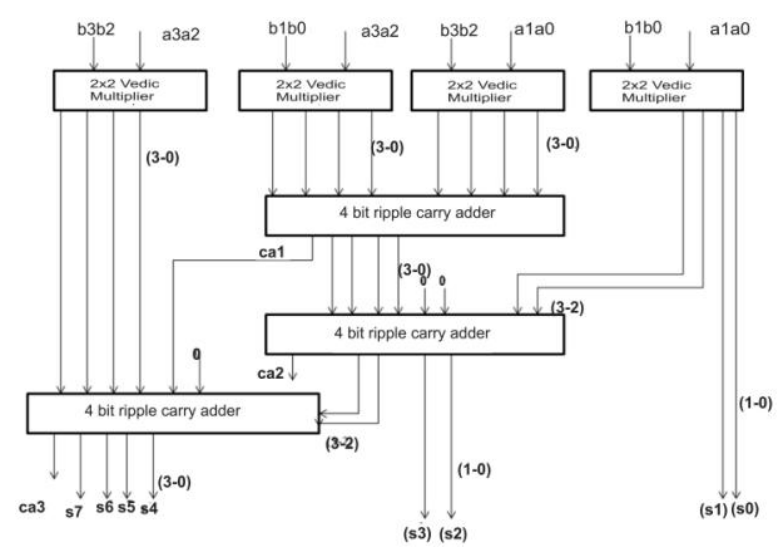

Fig. 5 Block Diagram of 4x4 bit Vedic Multiplier.

\subsection{Vedic Multiplier for $8 \times 8$ bit module.}

The $8 \times 8$ bit Vedic multiplier module as shown in block diagram in Fig. 6 can be easily implemented by using four $4 \times 4$ bit Vedic multiplier module. Let's analyze $8 \times 8$ multiplications say $A=A_{7} A_{6} A_{5} A_{4} A_{3} A_{2} A_{1} A_{0}$ and $\mathrm{B}=\mathrm{B}_{7} \mathrm{~B}_{6} \quad \mathrm{~B}_{5} \quad \mathrm{~B}_{4} \quad \mathrm{~B}_{3} \mathrm{~B}_{2} \mathrm{~B}_{1} \mathrm{~B}_{0}$. The output line for the multiplications result will be of 16 bits as $=\mathrm{S}_{15} \mathrm{~S}_{14} \mathrm{~S}_{13} \mathrm{~S}_{12} \mathrm{~S}_{11} \mathrm{~S}_{10} \mathrm{~S}_{9} \mathrm{~S}_{8} \mathrm{~S}_{7} \mathrm{~S}_{6} \mathrm{~S}_{5} \mathrm{~S}_{4} \mathrm{~S}_{3} \mathrm{~S}_{2} \mathrm{~S}_{1} \mathrm{~S}_{0}$. Lets divide $\mathrm{A}$ and $\mathrm{B}$ into two parts; say the 8 bit multiplicand $\mathrm{A}$ can be decomposed into pair of 4, bits AH-AL. Similarly multipli-cand B can be decomposed in BH-BL. The 16 Bit product can be written:

$\mathrm{P}=\mathrm{AxB}=(\mathrm{AH}-\mathrm{AL}) \mathrm{x}(\mathrm{BH}-\mathrm{BL})$

$=\mathrm{AHxBH}+(\mathrm{AHxBL}+\mathrm{ALxBH})+\mathrm{ALxB} \mathrm{L}$.

Using the fundamental of Vedic multiplication, taking four bits at a time and using 4 bit multiplier block we can perform the multiplication. The outputs of $4 \times 4$ bit multipliers are added accordingly to obtain the final product [11-13]. Here total three 8 bit Ripple-Carry Adders are required as shown in Fig 6.

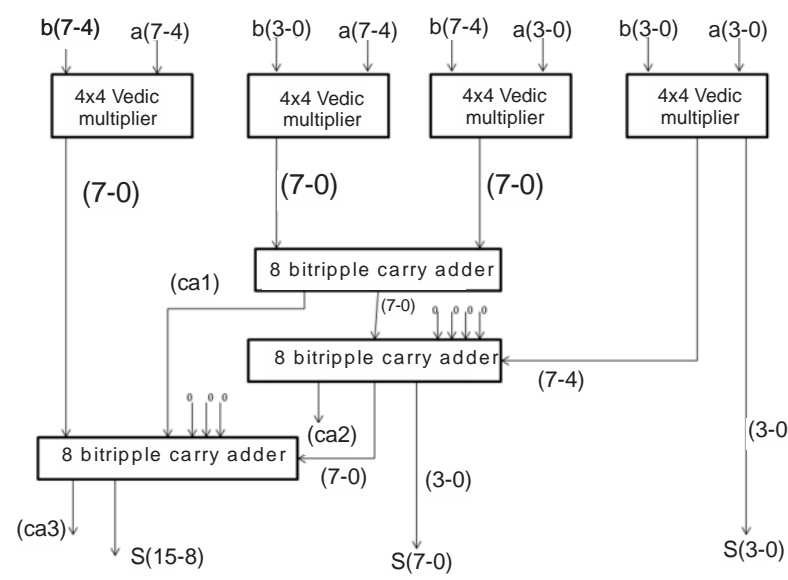

Fig 6: Block Diagram of 8x8 bit Vedic Multiplier.

\section{Design Verification and Implementation}

In this work, 8x8 bit Vedic multiplier is designed in VHDL (very High speed Integrated Circuits Hardware Description Language) Logic Synthesis and Simulation is done in Xilinx ISE 8.2 i Project Navigator and Isim simulator integrated in the Xilinx package. The perfor-mance of circuit is evaluated on the Xilinx device family, Spartan 2, XC2550 and package TQ144. The summary of the device description of the vertex FPGA used is explained in the table 1 
Table $1:$ Summary of FPGA Features

\begin{tabular}{|c|c|}
\hline Device Family & Vertex \\
\hline Device & XC 2550 \\
\hline Package & TQ 144 \\
\hline Speed Grade & -6 \\
\hline
\end{tabular}

\subsection{Simulation Results}

After the successful compilation the RTL view generated is shown in Fig. 7(a) and 7(b).

Fig. 8 and Fig. 9: show the simulation result of $8 \times 8$ bit Vedic multiplier for unsigned binary and decimal number respectively .

Fig 8 (a) and 8 (b) show the simulation result, when multiplicand Vedic_mult $8 \times 8 /$ multi is $=111111111$ (255) and the multiplier Vedic_mult $8 \times 8 / \mathrm{mplic}$ is=111111111 (255). The results of 16-bit output is obtained, Vedic_mult 8x8/p-out $=111111100000001(65025)$

Fig 9(a) and Fig 9(b) show the simulation result of binary and decimal respectively. The 8 bit operands are unsigned and the product of $000011111(15) \times 000011111(15)=0000000011100001(255)$.

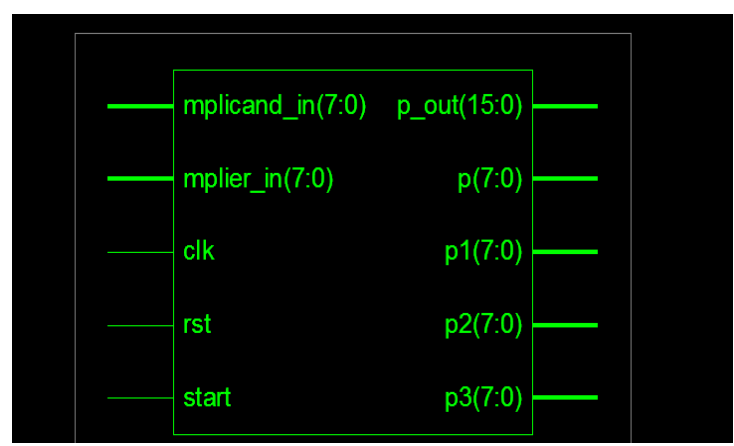

7(a)

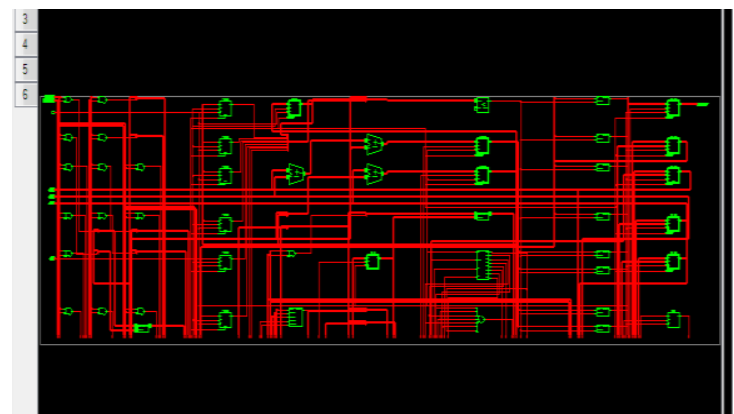

7(b)

Fig7 : RTL View of 8x8 bit Vedic Multiplier

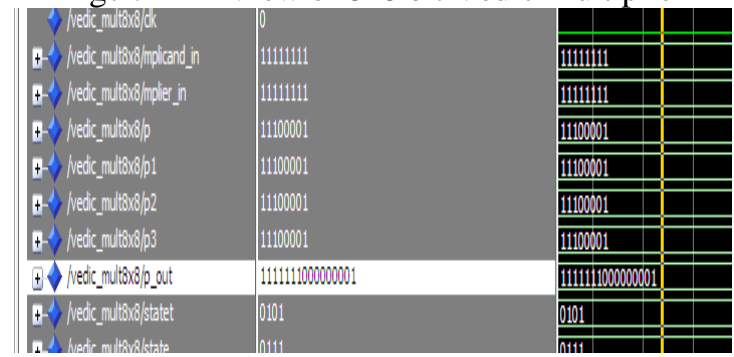

$8(a)$ 


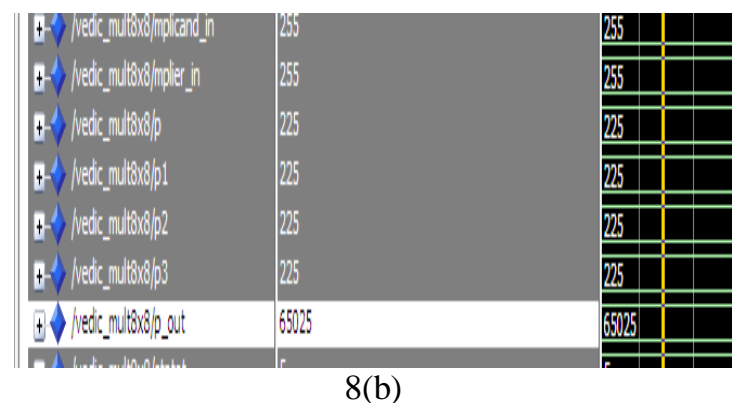

Fig. 8 Simulation waveforms of $8 \times 8$ bit Vedic Multiplier for multiplier $:$ For multiplicand $=11111111(255)$ and multiplier $=11111111(255)$

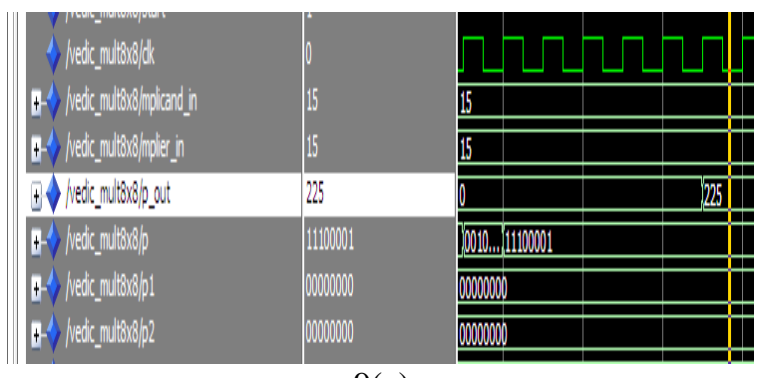

9(a)

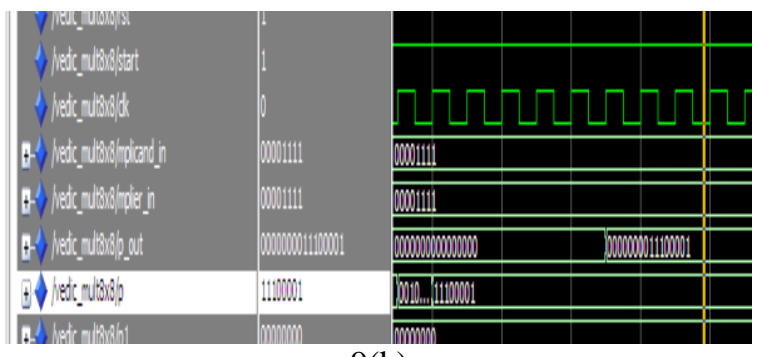

9(b)

Fig. 9 Simulation waveforms of $8 \times 8$ bit Vedic Multiplier for multiplier : For multiplicand $=00001111(15)$ and multiplier $=00001111(15)$

\subsection{Synthesis Results}

Table. 2 below shows the Synthesis report of the Vedic multiplier with the logic resource utilization.

Table . 2 Synthesis Result

\begin{tabular}{|l|l|c|c|}
\hline \multicolumn{4}{|c|}{ Device utilization Summary (estimated values) } \\
\hline Logic utilization & $\begin{array}{l}\text { use } \\
\text { d }\end{array}$ & $\begin{array}{l}\text { Availabl } \\
\text { e }\end{array}$ & Utilization \\
\hline Number of Slices & 174 & 192 & $90 \%$ \\
\hline Number of slice Flip Flop & 133 & 384 & $34 \%$ \\
\hline Number of 4 input LUTs ; & 326 & 384 & $84 \%$ \\
\hline Number of Bounded 10 Bs; & 67 & 90 & $77 \%$ \\
\hline Number of GCLKs & 1 & 4 & $25 \%$ \\
\hline
\end{tabular}

Synthesis was done using Xilinx ISE 8.21. The device chosen for synthesis is $2 \mathrm{~s} 15 \mathrm{cs} 144-6$. The Computation path delay for proposed $8 \times 8$ bit Vedic multiplier is found to be $8.460 \mathrm{~ns}$.

The power consumption was measured by using Xpower option available in project Navigator in ISE 6.1 Power consumption is $9.29 \mathrm{~mW}$. Total memory usage is 166744 Kilobytes.

\section{Conclusion}

This paper presents an efficient method of multiplication (UrdhvaTiryakbhyam) Sutra's based on Vedic mathematics. It gives us method for hierarchical multiplier design and clearly indicates the computational advantages offered by Vedic methods.

The Computational path delay for proposed $8 \times 8$ bit Vedic multiplier is found to be lesser then the conventional multiplier. Hence our motivation to reduce delay is finally fulfilled. Vedic multiplier has less 
number of gates required for given $8 \times 8$ bit multiplier so its power dissipation is very small as compared to other multiplier architecture. In terms of area also, the proposed multiplier is better than the conventional multiplier. An awareness of Vedic mathematics can be effectively increased if, it is included in engineering education which may lead to improvement significantly in many areas where fast arithmetic computational are critical such as real time DSP applications.

\section{References}

[1] Swami Bharati Krshna Tirthaji, Vedic Mathematics. (Delhi: Motilal Banarsidass Publishers, 1965).

[2] A. Haveliya "A Novel Design for High Speed Multiplier for Digital Signal Processing Applications (Ancient Indian Vedic mathematics approach", International Journal of Technology And Engineering System(IJTES), 2 (1), 2011.

[3] H. S. Dhillon and A. Mitra "A Digital Multiplier Architecture using Urdhava Tiryakbhyam Sutra oj Vedic Mathematics" IEEE Conference Proceedings, 2008.

[4] P. Mehta, D. Gawali "Conventional versus Vedic mathematical method for Hardware implementation of a multiplier" International Conference on Advances in Computing, Control, and Telecommunication Technologies, 2009.

[5] H. Thapliyal, S. Kotiyal and M.B. Srinivas, "Design and Analysis of a Novel Parallel Square and Cube Architecture Based on Ancient Indian Vedic Mathematics", Proceedings on 48th IEEE International Midwest Symposium on Circuits and Systems (MWSCAS), 2005.

[6] S. Akhtar, "VHDL Implementation of Fast $\mathrm{NxN}$ multiplier Base on Vedic Mathematics," Jaypee Institute of Information Technology University, Noida, 2011307 U.P, India, IEEE, 2007.

[7] L. Sriraman, T.N. Prabakar."Design and Implementation of two variable Multiplier using KCM and Vedic Mathematics", $1^{s t}$ International Conference on Recent Advances in Information Technology IEEE, 2012.

[8] P. Verma, K.K. Mehta, "Implementation of an efficient multiplier based on Vedic Mathematics using EDA Tool", International Journal of Engineering and Advance Technology (IJEAT) ISSN : 1 (5), 2012, 2249-8958.

[9] L. G. Moses. S and M Thilagar, "VLSI Implementation of high speed DSP algorithms using Vedic Mathematics." International Journal of Computers Communication and Information System. 2 (1), 2010, 0976 - 1349.

[10] B.C. Paul, F.S. Fujita., M. Okajima. "ROM Based logic (RBL) Design, A low- power 16 bit Multiplier", IEEE Journal of solid State Circuits, 44 (11), 2009, 2935-42.

[11] M Poornima, S. K. Patil, S. Kumar, K.P. Shridhar, H. Sanjay "Implementation of multiplier using Vedic Algorithm", International Journal of Innovative Technology and Exploring Engineering (IJITEE), ISSN : 2 (6), 2013, 2278-3075.

[12] K. Sethi, R. Panda, "An Improved squaring circuit for binary Numbers", International Journal of Advanced computer science and Application (IJACSA), 3 (2), 2012.

[13] H. Thapliyal, M. B. Srinivas and H. R. Arabnia, "Design And Analysis of a VLSI Based High Performance Low Power Parallel square Architecture", Proc. Int. Conf. Also. Math. Compo. Sc., Las Vegas, June 2005, 72-76.

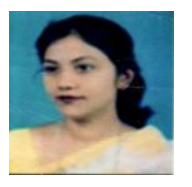

Ms. Anju is a M. Tech. scholar in VLSI Design at IMS Engineering Ghaziabad, affiliated to Mahamaya Technical University Noida.

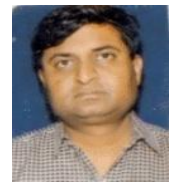

V.K. Agrawal is a senior faculty at IMS Engineering Ghaziabad \& is project guide of Ms. Anju. His interest of research is, VLSI Design \& Allied branches. He has published many research papers in International Journal \& Conferences. 